\title{
Multifocal ERG findings in carriers of X-linked retinoschisis
}

\author{
Linda S. Kim - William Seiple • \\ Gerald A. Fishman · Janet P. Szlyk
}

Received: 6 October 2006/ Accepted: 27 November 2006/ Published online: 19 December 2006

(C) Springer Science+Business Media B.V. 2006

\begin{abstract}
Purpose To determine whether retinal dysfunction in obligate carriers of $\mathrm{X}$-linked retinoschisis (XLRS) could be observed in local electroretinographic responses obtained with the multifocal electroretinogram (mfERG).

Methods Nine obligate carriers of XLRS (mean age, 46.2 years) were examined for the study. Examination of each carrier included an ocular examination and mfERG testing. For the mfERG, we used a 103-scaled hexagonal stimulus array that subtended a retinal area of approximately $40^{\circ}$ in diameter. The amplitudes and implicit times in each location for the mfERG were compared with the corresponding values determined for a group of 34 normally-sighted, age-similar control subjects.
\end{abstract}

L. S. Kim · G. A. Fishman (ه) · J. P. Szlyk Department of Ophthalmology and Visual Sciences (MC648), College of Medicine, University of Illinois at Chicago, Room 3.85, Eye and Ear Infirmary, 1855 West Taylor Street, Chicago, IL 60612-7234, USA e-mail: gerafish@uic.edu

\section{W. Seiple}

Department of Ophthalmology, New York University School of Medicine, New York, NY, USA

\section{W. Seiple $\cdot$ J. P. Szlyk}

Research and Development Service, Jesse Brown Veterans Administration Medical Center, West Side Division, Chicago, IL, USA
Results Mapping of 103 local electroretinographic response amplitudes and implicit times within a central $40^{\circ}$ area with the mfERG showed regions of reduced mfERG amplitudes and delayed implicit times in two of nine carriers.

Conclusions The mfERG demonstrated areas of retinal dysfunction in two carriers of XLRS. When present, retinal dysfunction was evident in the presence of a normal-appearing fundus. Multifocal ERG testing can be useful for identifying some carriers of XLRS.

Keywords Carriers - Multifocal electroretinography · Situs inversus · X-linked retinoschisis

\section{Introduction}

Juvenile $\mathrm{X}$-linked retinoschisis (XLRS) is a hereditary, vitreoretinal degeneration that was initially described in 1898 by Haas [1] and subsequently recognized to have an X-linked recessive inheritance in 1938 by Mann and Mac Rae [2]. It is characterized by decreased visual acuity within the first to second decade of life and cystic-appearing lesions within the fovea [3-5]. Approximately $50 \%$ of patients will also show peripheral retinoschisis [3-5]. A selective or predominant $b$-wave amplitude reduction on full-field electroretinogram (ERG) testing is a distinctive feature of the disease [6]. 
In other $\mathrm{X}$-linked diseases, such as $\mathrm{X}$-linked retinitis pigmentosa, choroideremia and $\mathrm{X}$-linked ocular albinism, because of the principle of Lyonisation, which predicts a random inactivation of one $\mathrm{X}$ chromosome within each cell, female carriers can express some features of the diseases [7-9]. As a rule, female carriers of XLRS do not exhibit any clinically apparent fundus abnormalities [3, 4]. Isolated observations of foveal cysticappearing lesions in female carriers from families with XLRS have been reported. There were six such female carriers noted out of a total of 13 who were examined in two different families with consanguineous marriages reported by two previous studies [10,11]. Foveal changes, described as wrinkling of the internal limiting membrane, of one eye was reported in one female XLRS carrier from a non-consanguineous marriage by Wu et al. [12]. One study did find that 11 out of 11 obligate carriers showed an abnormal rod-cone interaction by psychophysical testing [13]. Nevertheless, carrier detection of XLRS currently can most reliably be successful by identifying genetic mutations in a gene that codes for a protein referred to as retinoschisin (RS1) [14, 15]. In the current study, we evaluated the possible role of multifocal ERG (mfERG) testing as a means of detecting functional abnormalities in obligate carriers of XLRS.

\section{Methods}

Nine obligate carriers (eight parents, one offspring) of XLRS patients were enrolled in the study. They were selected on the basis of their availability and willingness to participate in the study. Each had at least one male family member who was diagnosed as having characteristic XLRS findings by one of the authors (GAF). Five of the nine XLRS patients were confirmed as having the causative gene RS1. None of the carriers had any other medical or ocular conditions that might have affected their retinal function. After each carrier was counseled regarding the study, they signed a written informed consent approved by the Institutional Review Board at the University of Illinois at Chicago. The examinations were conducted in accordance with Health Insurance Portability and Accountability Act regulations.

All carriers were examined at the Department of Ophthalmology, University of Illinois at Chicago by two of the authors (GAF, LSK). Bestcorrected visual acuity (BCVA) was measured in each carrier with a Snellen projection chart. A dilated fundus examination and slit-lamp biomicroscopy of the lens and anterior segment of both eyes were performed on all carriers as were mfERG (Electro-Diagnostic Imaging, San Mateo, CA) measurements. Multiple retinal areas were stimulated to record local cone responses using a stimulus array of 103-scaled hexagons subtending a retinal area of approximately $40^{\circ}$ in diameter. Each hexagon was scaled with eccentricity to obtain approximately equal amplitudes of local ERG responses [16]. The luminance of each hexagon was modulated according to a binary m-sequence. Individual stimulus elements were modulated between black $\left(0.45 \mathrm{~cd} / \mathrm{m}^{2}\right)$ and white $\left(280 \mathrm{~cd} / \mathrm{m}^{2}\right)$ for a time averaged luminance of $140.23 \mathrm{~cd} / \mathrm{m}^{2}$ (approximately $3.8 \mathrm{log} \mathrm{td}$ ). The surround luminance was set at $140 \mathrm{~cd} / \mathrm{m}^{2}$. The stimulus was displayed on a black-and-white monitor driven at a frame rate of $75 \mathrm{~Hz}$ (Nortec, Plymouth, MN). Each subject's vision was optimally corrected with a refractor/camera system for the fixed viewing distance of $40 \mathrm{~cm}$. To ensure equal magnification of the stimulus array, the distance between a subject's eye and the refractor/camera was adjusted for each subject to obtain a sharp image on a control monitor. Each carrier's pupil was dilated with $2.5 \%$ phenylephrine and $1 \%$ tropicamide to obtain a pupil diameter of at least $7 \mathrm{~mm}$. Multifocal ERGs were recorded only for the right eye for each of the XLRS carriers. A Burian-Allen bipolar contact lens electrode (Hansen Ophthalmic Laboratories, Iowa City, IA) was used and grounded to the ipsilateral ear. Before insertion of the contact lens electrode, a carrier's cornea was anesthetized with $0.5 \%$ proparacaine, and the left eye was occluded. The total recording time was approximately $8 \mathrm{~min}$ divided into 32 segments. All carriers were required to maintain fixation during each of $14 \mathrm{sec}$ segments. Segments with large eye movements, losses of fixation, or blinks were discarded and re-recorded. The raw data were 
Table 1 The age, visual acuity for the right eye (OD) and left eye (OS) and fundus findings for each of the X-linked retinoschisis carriers

\begin{tabular}{|c|c|c|c|c|}
\hline Carrier & $\begin{array}{l}\text { Age } \\
\text { (years) }\end{array}$ & $\begin{array}{l}\text { VA } \\
\text { OD }\end{array}$ & $\begin{array}{l}\text { VA } \\
\text { OS }\end{array}$ & Fundus findings \\
\hline 1 & 35 & $20 / 15$ & $20 / 15$ & Mild situs inversus OU \\
\hline 2 & 47 & $20 / 20$ & $20 / 20$ & Normal OU \\
\hline 3 & 23 & $20 / 20$ & $20 / 20^{-1}$ & Normal OU \\
\hline 4 & 53 & $20 / 20$ & $20 / 20$ & Mild situs inversus OU \\
\hline 5 & 48 & $20 / 25$ & $20 / 20$ & Situs inversus $\mathrm{OU}$ \\
\hline 6 & 58 & $20 / 20$ & $20 / 20$ & Situs inversus $\mathrm{OU}$ \\
\hline 7 & 39 & $20 / 20$ & $20 / 20$ & Mild situs inversus OU \\
\hline 8 & 43 & $20 / 20^{-2}$ & $20 / 20$ & $\begin{array}{l}\text { Situs inversus inferior } \\
\text { vein OS, anomalous } \\
\text { branching OS > OD }\end{array}$ \\
\hline 9 & 70 & $20 / 20^{-2}$ & $20 / 20^{-2}$ & $\begin{array}{c}\text { Anomalous branching } \\
\text { OU, situs inversus } \\
\text { superior artery OD, } \\
\text { inferior artery OS }\end{array}$ \\
\hline
\end{tabular}

filtered at a bandpass of 10 to $300 \mathrm{~Hz}$, amplified at a gain of 100,000 (Astro-Med/Gass model CP11 amplifier), and digitized at $1200 \mathrm{~Hz}$. Each local response was isolated by a cross correlation between the m-sequence and response cycle according to the VERIS algorithm.

The amplitude (a-scale) and implicit time (t-scale) of all local (first-order) mfERG responses were derived using the algorithm of Hood and $\mathrm{Li}$ [17]. For the current analysis, raw waveforms were exported from the VERIS 3.0 for each of the 34 (15 male, 19 female) age-similar (30-79 years) normally sighted control subjects. Artifact reject and averaging with neighbors were turned off.

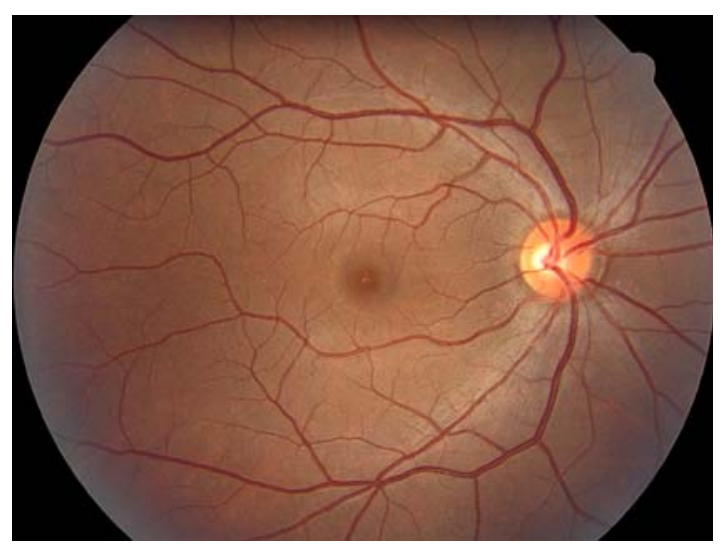

Fig. 1 Fundus photograph of XLRS carrier \#1 demonstrating situs inversus of the retinal veins
Template wave forms were constructed for each hexagon from the average of all the control subjects' data. These averaged templates were fitted using a least-squares fitting procedure to each control subject's data by stretching horizontally (timing) and vertically (amplitude). For each hexagon, the a-scale values were then averaged across all normal subjects and standard deviations calculated. This was also done separately for the t-scale values. Each carrier's data were then fit by the control templates and the resulting values for the a-scale and t-scale were plotted for each hexagon. For the a-scale, a number less than one indicates an amplitude lower than for the average control subjects and for the t-scale, a number greater than one indicates a timing delayed compared to the average control subjects. The statistical probability of each value was calculated using the control subjects mean and \pm 3 standard deviations (SD) for the a-scale and t-scale respectively and at each hexagon. The grey hexagons indicate $>3 \mathrm{SD}$ above the normal mean. For the $99.7 \%$ confidence level (grey hexagons) five control subjects had one abnormal amplitude and two subjects had two abnormal grey hexagons on the ascale. For implicit time, six control subjects had one abnormal grey hexagon and two had two abnormal grey hexagons. Therefore, for this analysis, any carrier exhibiting a total of three or more grey hexagons were considered abnormal. A black hexagon indicated that a measurable signal could not be detected from the noise level. None of the 34 control subjects had any hexagons that were depicted as black. We used a criterion of two or more black hexagons for a carrier as highly likely to represent a local abnormality.

\section{Results}

The nine XLRS carriers ranged in age from 2370 years, with a mean age of 46.2 years. All showed a normal exam of the anterior segment of the eye, including the lens. Visual acuity was $20 / 25$ or better in each eye. None showed any fundus abnormalities. These findings are summarized in Table 1. Seven of the nine obligate carriers were observed to have varying degrees, from mild to moderate, of situs inversus of their 

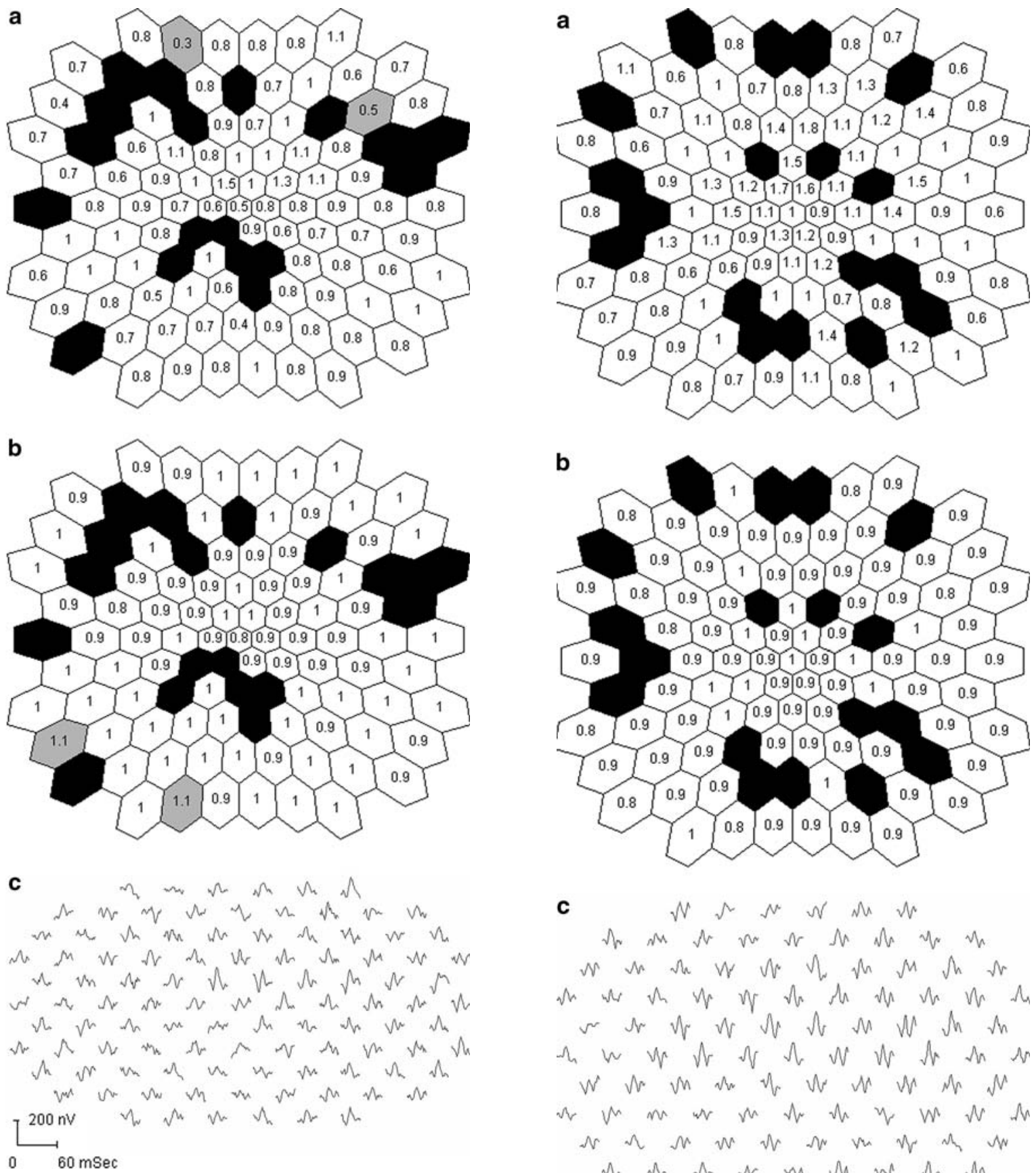

Fig. 2 Multifocal electroretinogram (mfERG) results of $\mathrm{X}$-linked retinoschisis (XLRS) carrier \#8 exhibiting a mosaic pattern of retinal dysfunction. MfERG amplitudes (a) and implicit times (b) for the right eye. In this figure, as in figure 3 , the white hexagons show regions falling within 2 standard deviations (SD) of the normal mean. The grey hexagons represent locations that are more than 3 SD from the normal mean while the black hexagons indicate a nonmeasurable response. MfERG waveform traces are illustrated below (c)

$$
\begin{aligned}
& \text { C }
\end{aligned}
$$

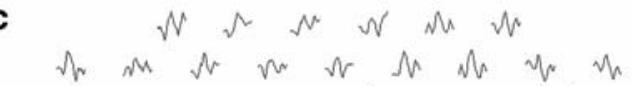

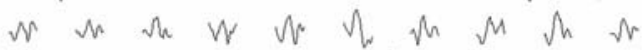

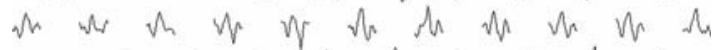

$$
\begin{aligned}
& \checkmark \text { or of of of } d \text { a W } M \text { in }
\end{aligned}
$$

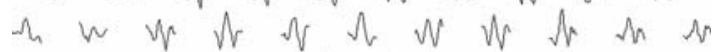

$$
\begin{aligned}
& W \text { ar } M \text { or of w } M \text { or } M \text { M }
\end{aligned}
$$

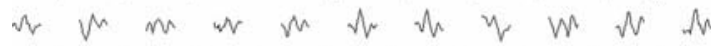

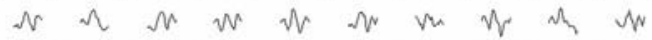

$$
\begin{aligned}
& \text { th in of w w W W A d a } \\
& 200 \mathrm{nv} M \text { a th o o d } \\
& 0 \quad 60 \mathrm{msec}
\end{aligned}
$$

Fig. 3 Multifocal electroretinogram (mfERG) results of XLRS carrier \#3 exhibiting a mosaic pattern of retinal dysfunction. MfERG amplitudes (a) and implicit times (b) for the right eye. MfERG waveform traces are illustrated below (c) 
temporal retinal vessels. In ocular situs inversus, the temporal vessels leave the optic disc directed towards the nasal retina before making a sharp temporal turn. The fundus photograph for Carrier \#1 with situs inversus is shown in Fig. 1. In a study of fundus findings for XLRS patients, situs inversus was observed in $32 \%$ of patients with XLRS [18]. To our knowledge, situs inversus of the retinal vessels has not been emphasized as a possible finding in carriers of XLRS.

Two of nine carriers demonstrated a mosaic pattern of statistically significant amplitude reductions (Figs. 2a, 3a) and implicit time delays (Figs. 2b, 3b). The actual mfERG tracings and hexagon analysis for these two carriers are shown in Figs. 2 and 3.

\section{Discussion}

Our findings support the conclusion that the presence of a functional abnormality on mfERG testing, in the absence of other causes for retinal dysfunction in a female at risk, should suggest the presence of a carrier state for XLRS, which could then be further confirmed by genetic testing. However, a normal mfERG result would not, by itself, rule out the carrier state. Piao and coworkers [19] showed that mfERG cone-mediated responses were more impaired centrally than in the more peripheral retina in their study of seven male patients with XLRS. They also observed a higher frequency of delayed implicit times than reduced amplitudes in their patients. We did not observe either of these features in our two carriers.

The presence of situs inversus of the retinal vessels in a female from a family with XLRS is also suggestive of the carrier state. Our findings on mfERG testing demonstrate the effect of Lyonisation on retinal function in carriers of XLRS. A similar finding on mfERG testing was observed in carriers of X-linked retinitis pigmentosa [20]. Multifocal ERG testing may be a useful technique for the detection and monitoring of localized retinal dysfunction in some carriers of XLRS.

Acknowledgments This study was supported by The Foundation Fighting Blindness, Owings Mills, Maryland, USA; The Grant Healthcare Foundation, Chicago Illinois,
USA; NIH core grant EY01792, Bethesda Maryland, USA; Rehabilitation Research and Development Service, US Department of Veterans Affairs, Washington DC, USA; and an unrestricted departmental grant from Research to Prevent Blindness, Inc., New York, New York, USA. The authors have no proprietary interest in this work.

\section{References}

1. Haas J (1898) Ueber das zusammenvorkommen von veränderungen der retina and chorioidea. Arch Augenheikd 37:343-348

2. Mann I, MacRae A (1938) Congenital vascular veils in the vitreous. Br J Ophthalmol 22:1-10

3. Deutman AF (1971) Sex-linked juvenile retinoschisis. In: Deutman AF (ed) The hereditary dystrophies of the posterior pole of the eye. Van Gorcum, Assen, The Netherlands pp 48-98

4. George NDL, Yates JRW, Moore AT (1995) X-linked retinoschisis. Br J Ophthalmol 79:697-702

5. Roesch MT, Ewing CC, Gibson AE et al (1998) The natural history of $\mathrm{X}$-linked retinoschisis. Can $\mathrm{J}$ Ophthalmol 33:149-158

6. Peachey NS, Fishman GA, Derlacki DJ et al (1987) Psychophysical and electroretinographic findings in Xlinked juvenile retinoschisis. Arch Ophthalmol 105:513-516

7. Krill AE (1969) X-chromosomal-linked diseases affecting the eye: status of the heterozygote female. Trans Am Ophth Soc 67:535-608

8. Jay B (1985) X-linked retinal disorders and the Lyon hypothesis. Trans ophthalmol Soc UK 104:836-844

9. Lyon MF (1962) Sex chromatin and gene action in the mammalian X chromosome. Am J Hum Genet 14:135148

10. Rodriguez FJ, Rodriguez A, Mendozo-Londono R et al (2005) $\mathrm{X}$-linked retinoschisis in three females from the same family: a phenotype-genotype correlation. Retina 25:69-74

11. Ali A, Feroze AH, Rizvi ZH et al (2003) Consanguineous marriage resulting in homozygous occurrence of $\mathrm{X}$-linked retinoschisis in girls. Am J Ophthalmol 136:767-769

12. Wu G, Cotlier E, Brodie S (1985) A carrier state of Xlinked juvenile retinoschisis. Ophthalmic Paediatr Genet 5:13-17

13. Arden GB, Gorin MB, Polkinghorne PJ et al (1988) Detection of the carrier state of X-linked retinoschisis. Am J Ophthalmol 105:590-595

14. Sauer CG, Gehrig A, Warneke-Wittstock R et al (1997) Positional cloning of the gene associated with X-linked juvenile retinoschisis. Nat Genet 17:164-170

15. Wang T, Zhou A, Waters CT et al (2006) Molecular pathology of $\mathrm{X}$-linked retinoschisis: mutations interfere with retinoschisin secretion and oligomerisation. Br J Ophthalmol 90:81-86

16. Sutter EE, Tran D (1992) The field topography of the ERG components in man - I. The photopic luminance response. Vis Res 32:433-466 
17. Hood DC, Li J (1997) A technique for measuring individual multifocal ERG records. In: Yager D (ed) Noninvasive assessment of the visual system. Trends in optics and photonics series; II. Optical Society of America, Washington, DC pp 33-41

18. Apushkin MA, Fishman GA, Rajagopalan AS (2005) Fundus findings and longitudinal study of visual acuity loss in patients with $\mathrm{X}$-linked retinoschisis. Retina 25:612-618
19. Piao C, Kondo M, Nakamura M et al (2003) Multifocal electroretinograms in $\mathrm{X}$-linked retinoschisis. Invest Ophthalmol Vis Sci 44:4920-4930

20. Vajaranant TS, Seiple W, Szlyk JP et al (2002) Detection using the multifocal electroretinogram of mosaic retinal dysfunction in carriers of X-linked retinitis pigmentosa. Ophthalmology 109:560-568 